\title{
Imbalance of energy and momentum source terms of the sea wave transfer equation for fully developed seas
}

\author{
G. V. Caudal \\ Université Versailles-St-Quentin; CNRS/INSU, UMR8190, Laboratoire Atmosphères, Milieux, \\ Observations Spatiales - LATMOS-IPSL 11 Boulevard d'Alembert, 78280 Guyancourt, France
}

Correspondence to: G. V. Caudal (gerard.caudal@latmos.ipsl.fr)

Received: 12 June 2012 - Published in Ocean Sci. Discuss.: 31 July 2012

Revised: 13 November 2012 - Accepted: 19 November 2012 - Published: 13 December 2012

\begin{abstract}
In the concept of full development, the sea wave spectrum is regarded as a nearly stationary solution of the wave transfer equation, where source and sink terms should be in balance with respect to both energy and momentum. Using a two-dimensional empirical sea wave spectral model at full development, this paper performs an assessment of the compatibility of the energy and momentum budgets of sea waves over the whole spectral range. Among the various combinations of model functions for wave breaking and wind source terms tested, not one is found to fulfill simultaneously the energy and momentum balance of the transfer equation. Based on experimental and theoretical grounds, wave breaking is known to contribute to frequency downshift of a narrow-banded wave spectrum when the modulational instability is combined with wave breaking. On those grounds, it is assumed that, in addition to dissipation, wave breaking produces a spectral energy flux directed toward low wavenumbers. I show that it is then possible to remove the energy and momentum budget inconsistency, and correspondingly the required strength of this spectral flux is estimated. Introducing such a downward spectral flux permits fulfilling both energy and momentum balance conditions. Meanwhile, the consistency between the transfer equation and empirical spectra, estimated by means of a cost function $\mathrm{K}$, is either improved or slightly reduced, depending upon the wave breaking and wind source terms chosen. Other tests are performed in which it is further assumed that wave breaking would also be associated with azimuthal diffusion of the spectral energy. This would correspondingly reduce the required downward spectral flux by a factor of up to 5, although it would not be able to remove it entirely.
\end{abstract}

\section{Introduction}

The ocean wave prediction models are based on solving the energy conservation equation (Gelci et al., 1957; WAMDI, 1988; Banner and Morison, 2010). The evolution of the spectral wave-energy density is understood as being governed by the combination of several different physical processes: the propagation of energy, the energy input by wind forcing, the dissipation of energy through viscosity and wave breaking, and the exchange of energy between different wave components interacting weakly among themselves. Abundant literature has been devoted to finding relevant parameterizations for the different source terms of the transport equation. These have been incorporated efficiently into the wave prediction models, producing quite satisfactory operational results. In that context, a fully developed ocean wave spectrum would be describable as a nearly stationary solution, in which the different terms of the energy transfer equation tend to balance each other, leading to greatly reduced wave evolution. It should be stressed however that the concept of full development is just a convenient vehicle for a mental experiment, but is not expected to describe a real situation since winds are always non-stationary and non-uniform.

In the context of a stationary (or quasi-stationary) state describing a fully developed sea, this paper explores the consistency of the energy and momentum budgets of the sea wave system. For this purpose, in Sect. 2 I will perform a test of the consistency of the integral energy and momentum equations in the framework of current parameterizations of the source and sink terms of the transport equation, and standard models of the fully developed directional spectrum. This will require taking into account not only the low wavenumber range 
for which the sea wave spectra are deduced from measurements by operational buoys, but also the high wavenumber range. To describe this high wavenumber region of the sea wave spectrum, empirical spectra were built based on other means, such as wave tank data (e.g. Jähne and Riemer, 1990), measurements based on arrays of height gauges (Donelan et al., 1985), stereophotogrammetric analysis (Banner et al., 1989), and radar reflectivity measurements at different radio frequencies. When the whole spectral range is considered, it will be shown that standard parameterization of the source terms does not allow satisfying simultaneously the energy and momentum integral transport equations. In addition to producing wave dissipation, another effect of wave breaking will then be proposed in Sect. 3 involving downward flux of spectral energy in the wavenumber space. It will be shown that within this assumption it is possible to solve the energy and momentum budget inconsistency, and correlatively the required strength of this downward spectral flux will be estimated.

\section{Energy and momentum budget of the sea wave spectral evolution}

\subsection{Spectral evolution equations integrated over wavenumber}

Let $F(k, \varphi)$ be the surface elevation spectrum of sea waves as a function of wavenumber magnitude $k$ and azimuth $\varphi$. The evolution of $F(k, \varphi)$ is governed by the basic transport equation involving a superposition of source terms, and is usually written in the following manner:

$$
\begin{aligned}
\frac{\partial F(k, \varphi)}{\partial t}+c_{\mathrm{g}} \cdot \nabla F & =\left(\frac{\partial F}{\partial t}\right)_{\mathrm{w}}+\left(\frac{\partial F}{\partial t}\right)_{\mathrm{visc}} \\
& +\left(\frac{\partial F}{\partial t}\right)_{\mathrm{br}}+\left(\frac{\partial F}{\partial t}\right)_{\mathrm{nl}},
\end{aligned}
$$

where $c_{\mathrm{g}}$ is wave group velocity, $(\partial F / \partial t)_{\mathrm{w}}$ represents the wind input term, $(\partial F / \partial t)_{\text {visc }}$ accounts for the damping of capillary waves due to water viscosity, and also includes a swell dissipation term related to friction with the atmosphere, as reported by several studies (Tolman, 2002; Ardhuin et al., 2009), as will be discussed below. The other terms of the right-hand side of Eq. (1a) are, respectively, the wave breaking dissipation term, and the resonant nonlinear wave-wave interaction term.

The case of a fully developed sea corresponds to a quasistationary situation in the open ocean where the wind has blown steadily over a sufficient time that the wave spectral density does not evolve anymore. In that ideal case, for any wavenumber $\mathrm{k}$ and azimuth $\varphi$, both $\nabla F$ and $\partial F(k, \varphi) / \partial t$ are expected to be equal to zero, and the four source terms of the right-hand side of Eq. (1a) balance each other, yielding

$$
\left(\frac{\partial F}{\partial t}\right)_{\mathrm{w}}+\left(\frac{\partial F}{\partial t}\right)_{\mathrm{visc}}+\left(\frac{\partial F}{\partial t}\right)_{\mathrm{br}}+\left(\frac{\partial F}{\partial t}\right)_{\mathrm{nl}} \approx 0
$$

In reality, the existence of a fully developed sea describable as a perfect stationary state has not been clearly established, and sea waves might still be growing for an old sea, although the net growth has by then become weak. For example, the input and dissipation integrated energy source terms obtained by Banner and Morison (2010) cancel out almost perfectly as the asymptotic state is approached, as can be seen from their Figure $4 \mathrm{~b}$, where the total integrated source term obtained for the oldest seas is reduced to less than $5 \%$ of the input and dissipation source terms taken separately.

Instead of Eq. (1b), it is liable to express the transport equation in terms of the wave energy spectrum $\Phi(k, \varphi)$ that is related to $F$ through:

$\Phi(k, \varphi)=\rho\left(\omega^{2} / k\right) F(k, \varphi)$.

It is also possible to express it in terms of the momentum spectrum $\mathbf{M}(k, \varphi)=\rho \omega F(k, \varphi)(\mathbf{k} / k)$. In those expressions $\rho$ is water density and $\omega$ is wave angular velocity. The momentum $\mathbf{M}$ carried by the waves is a vector quantity, but if we limit ourselves to fully developed situations, symmetry of the 2-D spectrum implies that only the along-wind component of the momentum will be different from zero after integration over wavenumber. Taking $\varphi=0$ for the wind direction, that component may be written at wavenumber $(k, \varphi)$ :

$M(k, \varphi)=\rho \omega F(k, \varphi) \cos \varphi$.

For a fully developed sea $(\partial F(k, \varphi) / \partial t \approx 0)$, integrating the transport equation for the wave energy spectrum over wavenumber, we obtain

$$
\begin{aligned}
& \int_{0}^{\infty} \int_{-\pi}^{\pi}\left[\left(\frac{\partial \Phi}{\partial t}\right)_{\mathrm{w}}+\left(\frac{\partial \Phi}{\partial t}\right)_{\mathrm{visc}}\right] k \mathrm{~d} k \mathrm{~d} \varphi+ \\
& \int_{0}^{\infty} \int_{-\pi}^{\pi}\left(\frac{\partial \Phi}{\partial t}\right)_{\mathrm{br}} k \mathrm{~d} k \mathrm{~d} \varphi+\int_{0}^{\infty} \int_{-\pi}^{\pi}\left(\frac{\partial \Phi}{\partial t}\right)_{\mathrm{nl}} k \mathrm{~d} k \mathrm{~d} \varphi=0 .
\end{aligned}
$$

The last term of the left-hand side of Eq. (4), which represents the nonlinear transfer of energy by resonant wavewave interactions, is described classically as resulting from four-wave interactions (Hasselmann, 1962, 1963) or threewave interactions (Valenzuela and Laing, 1972). In a general discussion about interaction symmetries involving wave particle analogy, Hasselmann et al. (1994) report that conservation of energy and conservation of momentum both apply for all wave-wave resonant interaction processes, including four-wave and three-wave interactions (this is not the case for wave action conservation, which holds for four-wave but not three-wave interactions). As a consequence, after integration over the wavenumber space the energy and momentum budget of resonant nonlinear interactions is zero. Therefore, the last term of the left-hand side of Eq. (4) is equal to zero, and similarly the same property applies for the momentum 
transport equation. One then gets both equations:

$$
\begin{aligned}
& \int_{0}^{\infty} \int_{-\pi}^{\pi}\left[\left(\frac{\partial \Phi}{\partial t}\right)_{\mathrm{w}}+\left(\frac{\partial \Phi}{\partial t}\right)_{\mathrm{visc}}\right] k \mathrm{~d} k \mathrm{~d} \varphi+ \\
& \int_{0}^{\infty} \int_{-\pi}^{\pi}\left(\frac{\partial \Phi}{\partial t}\right)_{\mathrm{br}} k \mathrm{~d} k \mathrm{~d} \varphi=0 \\
& \int_{0}^{\infty} \int_{-\pi}^{\pi}\left[\left(\frac{\partial M}{\partial t}\right)_{\mathrm{w}}+\left(\frac{\partial M}{\partial t}\right)_{\mathrm{visc}}\right] k \mathrm{~d} k \mathrm{~d} \varphi+ \\
& \int_{0}^{\infty} \int_{-\pi}^{\pi}\left(\frac{\partial M}{\partial t}\right)_{\mathrm{br}} k \mathrm{~d} k \mathrm{~d} \varphi=0 .
\end{aligned}
$$

\subsection{Tests based on standard source terms for fully developed seas}

In order to check the integral energy and momentum budgets of Eqs. (5a) and (5b), it is necessary to quantify the different source terms involving wind input, viscosity and wave breaking. For each of them, we may define the rate of evolution of the sea wave spectrum $\beta_{\mathrm{sub}}(k, \varphi)$ according to

$\beta_{\text {sub }}(k, \varphi)=\frac{1}{F(k, \varphi)}\left(\frac{\partial F(k, \varphi)}{\partial t}\right)_{\text {sub }}$,

where the subscript "sub" refers to the type of source term involved ("visc" for viscous damping, "w" for wind input and "br" for wave breaking).

As concerns the wind input and wave breaking terms, they have been the subjects of numerous parameterizations in the literature. I tested different combinations of wind input and wave breaking models. Basic types of models for source terms will be described below (Sect. 2.2.2 and 2.2.3), and will be used to check the energy and momentum budgets. Although not exhaustive, these illustrate the main assumptions taken by standard models. Other source functions tested did not lead to qualitatively different conclusions, and did not allow removing the systematic trends obtained and described below.

\subsubsection{Viscous damping term}

The viscous damping term is expressed as

$\beta_{\text {visc }}=\beta_{\text {capil }}+\beta_{\text {swell }}$.

In Eq. (7), $\beta_{\text {capil }}$ is the damping term of capillary waves due to the viscosity of water, and is taken from Lamb (1932) as $\beta_{\text {capil }}=-4 v_{\mathrm{w}} k^{2}$, where $\nu_{\mathrm{w}}=1.3 \times 10^{-6} \mathrm{~m}^{2} \mathrm{~s}^{-1}$ is the kinematic viscosity of water. According to Dore (1978), however, the viscosity of the air gives a stronger dissipation for wavelength larger than $0.85 \mathrm{~m}$. Swell dissipation was observed by Tolman (2002) and Ardhuin et al. (2009), who found it to be consistent with the effects of friction with the atmosphere. The term $\beta_{\text {swell }}$ in Eq. (7) accounts for such damping of swells by friction with the air. Ardhuin et al. (2010) propose the following expressions for this swell dissipation term:

$\beta_{\text {swell }}=-1.2 \frac{\rho_{\mathrm{a}}}{\rho_{\mathrm{w}}}\left[2 k \sqrt{2 v_{\mathrm{a}} \omega}\right] \quad$ for $R e \leq R e_{\mathrm{c}}$

$\beta_{\mathrm{swell}}=-\frac{\rho_{\mathrm{a}}}{\rho_{\mathrm{w}}}\left[16 f_{\mathrm{e}} \omega^{2} u_{\mathrm{orb}} / g\right]$ for $R e>R e_{\mathrm{c}}$.

In these expressions, $R e=4 u_{\mathrm{orb}} a_{\mathrm{orb}} / \nu_{\mathrm{a}}$ is the boundary Reynolds number, where $u_{\text {orb }}$ and $a_{\text {orb }}$ are the significant surface orbital velocity and displacement amplitudes, and $v_{\mathrm{a}}=14 \times 10^{-6} \mathrm{~m}^{2} \mathrm{~s}^{-1}$ is the air viscosity. Following Ardhuin et al. (2010), the critical Reynolds number value $R e_{\mathrm{c}}$ is estimated as a function of significant wave height $H_{\mathrm{s}}$ according to $R e_{\mathrm{c}}=\left(2 \times 10^{5} \mathrm{~m}\right) / H_{\mathrm{s}}$, and the coefficient $f_{\mathrm{e}}$ is taken as

$f_{\mathrm{e}}=0.8\left[0.003+(0.015-0.018 \cos \varphi) \frac{u_{*}}{u_{\mathrm{orb}}}\right]$.

\subsubsection{Wind input source terms}

Among the numerous parameterizations for the atmospheric wind-related growth rate $\beta_{\mathrm{w}}$ found in the literature, some are based on the friction velocity $u_{*}$, others are based on the wind velocity at a given height, and still others are based on the velocity at a height scaled with the wavelength. Plant (1982) proposed

$\beta_{\mathrm{w}}(k, \varphi)=0.04 \cos \varphi\left(\frac{u_{*}}{c(k)}\right)^{2} \omega$,

where $c(k)$ is the wave phase speed, and $\omega$ is wave angular velocity.

On the basis on previous studies (Snyder et al., 1981; Komen et al., 1984), the WAMDI group (1988) used a linear rather than quadratic dependence for $\beta_{\mathrm{w}}$ for their third generation wave prediction model:

$\beta_{\mathrm{w}}(k, \varphi)=\max \left\{0,0.025 \frac{\rho_{\mathrm{a}}}{\rho_{\mathrm{w}}}\left(28 \frac{u_{*}}{c} \cos \varphi-1\right)\right\} \omega$,

where $\rho_{\mathrm{a}}$ and $\rho_{\mathrm{w}}$ are air and water densities, respectively.

Arguing that the appropriate reference wind is rather the wind at some height above the roughness elements that is related to their scale, Donelan and Pierson (1987) proposed a parameterization based upon the wind speed at a height $\pi / k$ :

$\beta_{\mathrm{w}}=0.194 \frac{\rho_{\mathrm{a}}}{\rho_{\mathrm{w}}} \omega\left[\max \left\{\left(\frac{U(\pi / k)}{c(k)} \cos \varphi-1\right), 0\right\}\right]^{2}$.

Following previous authors (Belcher and Hunt, 1993; Hara and Belcher, 2002), Kukulka and Hara (2005) proposed a wind input wave growth formulation under the sheltering 
assumption. According to this approach, the wave-induced stress of longer waves reduces the turbulent stress felt by shorter waves. The resulting $\beta_{\mathrm{w}}$ can be expressed as follows:

$\beta_{\mathrm{w}}(k, \varphi)=\frac{c_{\beta}(k) \omega}{\rho_{\mathrm{w}} c^{2}} h_{\beta}(\varphi) \tau_{\mathrm{o}}$

$\exp \left[-\int_{k^{\prime}=0}^{k} \int_{\varphi^{\prime}=-\pi / 2}^{\pi / 2} c_{\beta}\left(k^{\prime}\right) k^{\prime 3} h_{\beta}\left(\varphi^{\prime}\right) F\left(k^{\prime}, \varphi^{\prime}\right) \cos \varphi^{\prime} \mathrm{d} k^{\prime} \mathrm{d} \varphi^{\prime}\right]$,

where $h_{\beta}(\varphi)=\left[\max (\cos \varphi, 0]^{2}, c_{\beta}(k)\right.$ is a coefficient that depends upon the boundary Reynolds number $R e$, and $\tau_{\mathrm{o}}=$ $\rho_{\mathrm{a}} u_{*}^{2}$ is the total momentum flux.

In the capillary-gravity range, waves are steep and they may result in modification of the airflow, which will reduce the momentum flux from air to water. This phenomenon was studied by Janssen (1991) who proposed a quasi-linear theory resulting in an effective high wavenumber cutoff. More recently, Banner and Morison (2010) and Ardhuin et al. (2010) proposed a wave prediction model based on this approach, including slight tuning modifications. The parameterization of the wind input term $\beta_{\mathrm{w}}$ based on the works by Janssen (1991), Banner and Morison (2010), and Ardhuin et al. (2010), will be referred to as the JBA model, and is described in more detail in Appendix A.

I tested successively those 5 different wind input source terms, taken respectively from Plant (1982), Komen et al. (1984), Donelan and Pierson (1987), Kukulka and Hara (2005), and the JBA model. In all cases the total atmospheric term (including wind input and swell dissipation) was set to 0 for $\cos \varphi<0$.

\subsubsection{Wave breaking source terms}

Several approaches have been used for modeling $\beta_{\mathrm{br}}$. The first one considers that, even though wave breaking is a nonlinear process, the phenomenon applied to the sea waves is weak in the mean, and as a consequence it leads to a quasilinear behavior of the dissipation $\left(\frac{\partial \Phi}{\partial t}\right)_{\mathrm{br}}$ with respect to wave spectrum $\Phi$ (Hasselmann, 1974). This approach was followed by Komen et al. (1984), who proposed

$\beta_{\mathrm{br}}(k, \varphi)=-\gamma_{\mathrm{K}} P_{\mathrm{K}}(k, \varphi)$

with

$P_{\mathrm{K}}(k, \varphi)=\frac{\omega^{2}}{\langle\omega\rangle}$,

where $\gamma_{\mathrm{K}}$ is a non-dimensional constant, and $\langle\omega\rangle$ is mean angular frequency.

Another type of approach was proposed by Phillips (1985), who explored the consequences of the assumption that the processes of energy input from the wind, spectral flux divergence due to resonant nonlinear interactions, and loss by breaking are all important in the equilibrium range. These considerations led him to propose the following formulation for the variation of the wave action density $N(k, \varphi)=(g / \omega) F(k, \varphi)$ due to wave breaking:

$\left(\frac{\partial N}{\partial t}\right)_{\mathrm{br}}=-g k^{4} a B^{3}(k, \varphi)$,

where $g$ is acceleration of gravity, $B(k, \varphi)=k^{4} F(k, \varphi)$ is the saturation spectrum, and $a$ is a non-dimensional constant. In terms of $\beta_{\mathrm{br}}$, this can be rewritten as

$\beta_{\mathrm{br}}(k, \varphi)=-\gamma_{\mathrm{P}} P_{\mathrm{P}}(k, \varphi)$

with

$P_{\mathrm{P}}(k, \varphi)=\omega k^{8} F^{2}(k, \varphi)$

and $\gamma_{\mathrm{P}}$ is a non-dimensional constant.

More recently, due to the observed strong threshold behavior reported by Banner et al. (2002) for the wave breaking probability in the spectrum, threshold-based formulations for the breaking component of the dissipation term $\beta_{\mathrm{br}}$ were proposed (Banner and Morison, 2010; Ardhuin et al., 2010). The model results closely reproduced the observed breaking wave properties and wave spectral evolution. Ardhuin et al.'s (2010) approach will be expressed here as

$\beta_{\mathrm{br}}(k, \varphi)=-\gamma_{\mathrm{a}} P_{\mathrm{a}}(k, \varphi)$,

where $\gamma_{\mathrm{a}}$ is a non-dimensional constant, and $P_{\mathrm{a}}(k, \varphi)$ is given in Appendix B.

Similarly to what has been done for wind input source terms, I tested successively those 3 different wave breaking source terms taken respectively from Komen et al. (1984), Phillips (1985), and Ardhuin et al. (2010).

\subsubsection{Empirical sea wave spectral model}

In order to compute the integral quantities of Eqs. (5a) and (5b), one needs to use a model for the sea wave spectrum based on observations. As concerns the omnidirectional spectrum, I used the empirical sea wave spectral model by Elfouhaily et al. (1997) for different conditions of wind speed, assuming fully developed situations. An advantage of this model is that it describes the wave spectrum over the whole range of wavenumbers - from the spectral peak to capillary waves - on a purely empirical basis. Also, while this model was tuned on in situ observations of wave spectra performed both in ocean and in laboratory settings, it is also consistent with the optical mean square slope measurements by Cox and Munk (1954).

As concerns the directional behavior of the spectrum, observations have shown that it exhibits distinct directionally bimodal peaks (Young et al., 1995; Long and Resio, 2007). Resio et al. (2011) have proposed wave-age dependent selfsimilar bimodal model functions for the directional spreading consistent with recent observations. They showed that, 
with their model function, the nonlinear wave-wave interactions produce relatively constant fluxes of both energy and momentum through the equilibrium range. For those reasons I used Resio et al.'s (2011) directional spreading model function, assuming full development. This spreading function was used up to wavenumber $k=25 k_{\mathrm{p}}$, i.e. frequency $f=5 f_{\mathrm{p}}$, where $k_{\mathrm{p}}$ and $f_{\mathrm{p}}$ are the peak wavenumber and frequency, which corresponds roughly to the domain where Resio et al.'s model was validated against data. For higher wavenumbers, most directional observations are based upon remote sensing techniques (radar or optical observations including Banner et al.'s (1989) stereo-photogrammetric analysis), and give essential information about the centrosymmetric (or folded) spreading function. Although this approach is relevant for electromagnetic modelers, it is not appropriate here, since the transport equations written above must involve directional instead of folded spectra, and some hypothesis needs to be used to obtain a directional spectrum. For wavenumbers above $25 k_{\mathrm{p}}$, I used Elfouhaily's folded spreading function. Elfouhaily et al. define a delta ratio given by $\Delta(k)=(F(k, 0)-F(k, \pi / 2) /(F(k, 0)+F(k, \pi / 2))$. Following Donelan et al. (1985), I assumed a directional spectrum given by an hyperbolic secant spreading function $F(k, \varphi)=$ $F(k, 0) \operatorname{sech}^{2}(h \varphi)$, and the value of $h$ was adjusted in such a way that the resulting folded spectrum yields the delta ratio of Elfouhaily et al.'s model.

The momentum budget depends in a sensitive manner on the high wavenumber part of the spectrum, and it is therefore useful to estimate how much the momentum budget is sensitive to small errors in the high frequency part of the spectrum. For this purpose, tests were also performed using a composite spectrum where the model described above was replaced by Kudryavtsev et al.'s (2003) directional spectrum for high wavenumbers $\left(k \geq 25 k_{\mathrm{p}}\right)$. Elfouhaily et al.'s (1997) spectrum in the high wavenumber range was designed by using only laboratory data, as well as optical data at sea, purposefully excluding radar data. On the contrary, Kudryavtsev et al.'s (2003) spectrum was essentially based on normalized radar cross section (NRCS) measurements at sea, and more recent measurements of radar derived anisotropy of the sea surface mean square slope (Hauser et al., 2008) were found to be reasonably well fitted by Kudryavtsev et al.'s (2003) spectrum. As an example, a comparison between the two model spectra can be seen in Fig. 1 for a $10 \mathrm{~m} \mathrm{~s}^{-1}$ wind speed. In order to make the comparison easier, the curvature spectra $B(k, \varphi)=k^{4} F(k, \varphi)$ are plotted for two azimuthal directions $\varphi=0$ and $\varphi=\pi / 2$.

\subsubsection{Energy and momentum budgets integrated over wavenumber}

The wind input and wave breaking source terms described above, together with the viscous term (Eq. 7), were applied to the empirical sea wave spectrum in order to check the energy and momentum budgets expressed in Eqs. (5a) and (5b),

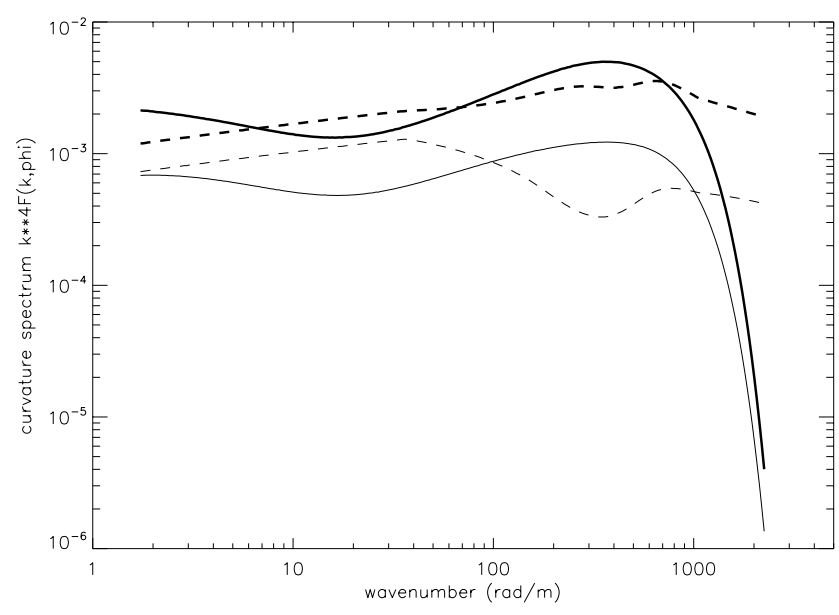

Fig. 1. Curvature spectrum $B(k, \varphi)=k^{4} F(k, \varphi)$ in the high wavenumber range ( $k \geq 25 k_{\mathrm{p}}$ ) as given by Elfouhaily et al.'s (1997) model (solid lines) and by Kudryavtsev et al.'s (2003) model (dashed lines), plotted for a $10 \mathrm{~m} / \mathrm{s}$ wind speed. For each model spectrum, the thick line stands for the spectrum along the wind direction $(\varphi=0)$, while the thin line stands for the spectrum in the crosswind direction $(\varphi=\pi / 2)$.

which may be rewritten

$$
\begin{aligned}
& \int_{0}^{\infty} \int_{-\pi}^{\pi}\left[\beta_{\mathrm{w}}+\beta_{\mathrm{visc}}\right] \Phi k \mathrm{~d} k \mathrm{~d} \varphi=\int_{0}^{\infty} \int_{-\pi}^{\pi} \beta_{\mathrm{br}} \Phi k \mathrm{~d} k \mathrm{~d} \varphi \\
& \int_{0}^{\infty} \int_{-\pi}^{\pi}\left[\beta_{\mathrm{w}}+\beta_{\mathrm{visc}}\right] M k \mathrm{~d} k \mathrm{~d} \varphi=\int_{0}^{\infty} \int_{-\pi}^{\pi} \beta_{\mathrm{br}} M k \mathrm{~d} k \mathrm{~d} \varphi .
\end{aligned}
$$

The expressions for the wave breaking or wind input source terms given in Sect. 2.2.2 and 2.2.3 should be regarded as giving functional forms, but a fine tuning of their relative amplitudes might be needed in order to fulfill the energy and momentum balance Eqs. (18a) and (18b). To perform this fine tuning of the relative amplitudes of the wind input and wave breaking source terms, I considered coefficient $\gamma$ of the wave breaking (referred as $\gamma_{\mathrm{k}}, \gamma_{\mathrm{p}}$, or $\gamma_{\mathrm{b}}$ in Eq. (14a), (16a), or (17), respectively) as an adjustable coefficient. Since both energy and momentum balance (18a and 18b) must be fulfilled, there are indeed two ways to perform the tuning of the same coefficient $\gamma$ :

$$
\gamma_{1}=\frac{\int_{0}^{\infty} \int_{-\pi}^{\pi}\left(\beta_{\mathrm{w}}+\beta_{\mathrm{visc}}\right) \Phi k \mathrm{~d} k \mathrm{~d} \varphi}{\int_{0}^{\infty} \int_{-\pi}^{\pi} P(k, \varphi) \Phi k \mathrm{~d} k \mathrm{~d} \varphi}
$$


Table 1. Ratio $R=\gamma_{2} / \gamma_{1}$, where $\gamma_{1}$ and $\gamma_{2}$ are the coefficients of the wave breaking dissipation term required to fulfill the energy and momentum balance, respectively, for different combinations of the expressions of $\beta_{\mathrm{w}}$ and $\beta_{\mathrm{br}}$ assumed. The sea wave spectral model for $k<25 k_{\mathrm{p}}$ is from Elfouhaily et al. (1997), with directional spreading function by Resio et al. (2011). Above $k=25 k_{\mathrm{p}}$, the sea wave directional spectral model is from Elfouhaily et al. (1997). A wind speed $U=10 \mathrm{~m} \mathrm{~s}^{-1}$ is assumed.

\begin{tabular}{lccc}
\hline Ratio $R=\gamma_{2} / \gamma_{1}$ & $\begin{array}{c}\beta_{\text {br Komen }} \\
\text { et al. (1984) }\end{array}$ & $\begin{array}{c}\beta_{b r} \text { Phillips } \\
(1985)\end{array}$ & $\begin{array}{c}\beta_{\text {br Ardhuin }} \\
\text { et al. (2010) }\end{array}$ \\
\hline$\beta_{\mathrm{w}}$ Plant (1982) & 2.97 & 2.55 & 2.67 \\
$\beta_{\mathrm{w}}$ WAMDI (1988) & 2.66 & 2.29 & 2.40 \\
$\beta_{\mathrm{w}}$ Donelan-Pierson (1987) & 5.11 & 4.39 & 4.60 \\
$\beta_{\mathrm{w}}$ Kukulka-Hara (2005) & 4.78 & 4.10 & 4.30 \\
$\beta_{\mathrm{w}}$ JBA model & 3.68 & 3.16 & 3.31 \\
\hline
\end{tabular}

$\gamma_{2}=\frac{\int_{0}^{\infty} \int_{-\pi}^{\pi}\left(\beta_{\mathrm{w}}+\beta_{\mathrm{visc}}\right) M k \mathrm{~d} k \mathrm{~d} \varphi}{\int_{0}^{\infty} \int_{-\pi}^{\pi} P(k, \varphi) M k \mathrm{~d} k \mathrm{~d} \varphi}$,

where $P(k, \varphi)$ is equal to $P_{\mathrm{k}}(k, \varphi), P_{\mathrm{p}}(k, \varphi)$, or $P_{\mathrm{a}}(k, \varphi)$ according to whether Eqs. (14a), (16a), or (17) is used. Of course, one expects both obtained values $\gamma_{1}$ and $\gamma_{2}$ to be close to each other, since they represent two estimates of the same quantity $\gamma$. Table 1 displays the ratio $R=\gamma_{2} / \gamma_{1}$ for the various combinations of the wind and breaking source terms described above. While the expected value of $R$ is $R=1$, one can see that there is a considerable scatter between the values of $R$ obtained, with a mean of $R=3.5$, and extreme values from 2.66 to 5.11 , depending on the functional form chosen for $\beta_{\mathrm{w}}$ and $\beta_{\mathrm{br}}$.

The computations of coefficients $\gamma_{1}$ and $\gamma_{2}$ depend not only on the functional forms of the source terms $\beta_{\mathrm{w}}$ and $\beta_{\mathrm{br}}$, but also on the assumed shape of the sea wave spectrum in the $(k, \varphi)$ space. The ratio $R$ (not shown) computed when replacing Resio et al.'s (2011) spreading function by other spreading functions (Elfouhaily et al., 1997; Donelan and Pierson, 1987; Banner, 1990) at low wavenumbers did not allow reduction of the ratio $R$ to a value close to 1 .

The ratio $R$ was also computed with the composite model mentioned in Sect. 2.2.4, where the spectrum based on Elfouhaily et al. (1997) and Resio et al. (2011) is replaced by Kudryavtsev et al.'s (2003) spectrum at high wavenumbers. The results obtained with this composite spectral model are given in Table 2. It can be seen that the new ratio $R$ is modified, but still remains within $35 \%$ of the values listed in Table 1 for each combination of the $\beta_{\mathrm{w}}$ and $\beta_{\mathrm{br}}$ models. The smallest value for $R(R=1.72)$ is obtained with Komen et al.'s (1984) model for $\beta_{\mathrm{br}}$, WAMDI's (1988) model for $\beta_{\mathrm{w}}$, and the composite spectral model used for Table 2. However, no combination of the current models of sea wave spectrum, wind input source term, and wave breaking source term was
Table 2. Same as Table 1, but Elfouhaily et al.'s (1997) wave spectrum is replaced by Kudryavtsev et al.'s (2003) spectrum at high wavenumbers $\left(k \geq 25 k_{\mathrm{p}}\right)$.

\begin{tabular}{lccc}
\hline Ratio $R=\gamma_{2} / \gamma_{1}$ & $\begin{array}{c}\beta_{\text {br Komen }} \\
\text { et al. (1984) }\end{array}$ & $\begin{array}{c}\beta_{b r} \text { Phillips } \\
(1985)\end{array}$ & $\begin{array}{c}\beta_{\text {br }} \text { Ardhuin } \\
\text { et al. (2010) }\end{array}$ \\
\hline$\beta_{\mathrm{W}}$ Plant (1982) & 2.58 & 3.32 & 3.45 \\
$\beta_{\mathrm{w}}$ WAMDI (1988) & 1.72 & 2.21 & 2.30 \\
$\beta_{\mathrm{w}}$ Donelan-Pierson (1987) & 4.00 & 5.15 & 5.36 \\
$\beta_{\mathrm{w}}$ Kukulka-Hara (2005) & 3.38 & 4.35 & 4.53 \\
$\beta_{\mathrm{w}}$ JBA model & 2.76 & 3.55 & 3.69 \\
\hline
\end{tabular}

indeed found to be able to produce a ratio $R$ equal (or at least close) to 1 .

Assuming, for example, that the wave breaking coefficient $\gamma$ (i.e. $\gamma_{\mathrm{k}}, \gamma_{\mathrm{p}}$, or $\gamma_{\mathrm{b}}$ of Eqs. 14a, 16a, or 17) is determined by the energy balance equation (i.e. $\gamma=\gamma_{1}$ ), the preceding results indicate that the wave momentum removed from the waves by wave breaking and viscous dissipation is unable to balance the momentum brought by the wind (this would require $\gamma=\gamma_{2}$, while instead $\gamma=\gamma_{1}<\gamma_{2}$ ). Thus, within the classical expressions for wave growth terms of wind input and wave breaking, and for fully developed situations, a proportion of about one half or more of the momentum brought by the wind to the waves would accumulate within the wave system. This is not a tenable assumption for a fully developed sea that is supposed to be nearly stationary.

\section{A modified formulation of the breaking source term restoring energy and momentum balance}

Due to the difficulty reported in the preceding section to fulfill both energy and momentum budgets, it appears that present parameterizations of the source terms are inconsistent with empirical spectral shapes. Does this mean that the chosen spectral shapes are unrealistic, or that the source term parameterizations are unrealistic? It is unfortunately not possible to answer this question. However, the spectral models were built by direct confrontation to the observations, taking advantage of numerous kinds of instruments (in situ as well as remote sensing data) operating in the various sea state conditions, and it would be uncomfortable to modify those purely observation-based wave spectra just in order to conform to the energy and momentum budget requirements. In contrast, the source terms of the transport equation are quantities determined in a more indirect manner (and for that reason, as seen in Sect. 2, numerous different approaches have been followed to parameterize those source terms). In view of these considerations, in this paper I choose to consider the empirical spectra as the observational reference. Basically, a way to restore energy and momentum balance would be to add an extra source term at low wavenumbers, and/or to add an extra sink term at high wavenumbers, but obviously there is not a unique way to modify the source terms in order to 
fulfill energy and momentum balance, and also such a modification should not alter significantly the resulting sea wave spectrum.

Among the source or sink terms, the one which is the least well established is the wave breaking term, because the processes represented by this unique term are indeed highly nonlinear, involving spilling of the crests of large gravity waves, formation and dissipation of whitecaps, spray, entrainment of air bubbles within the water upper layers, and production of turbulence at shorter scales. Also, since the wave breaking events occurring near the steep crests are localized features in the spatial domain, duality of the spectral analysis implies that their signature cannot be local in the wavenumber space. Thus, expressing $\beta_{\mathrm{br}}$ as a mere function of $F(k, \varphi)$ and $k$ may be considered as an oversimplification, even if it is scaled by integral quantities of the wave spectrum (as in Eq. 14b, for instance).

From the discussion above, it follows that one approach to restore energy and momentum balance would be to decrease the amplitude of the wave breaking sink term at low wavenumbers and to increase it at high wavenumbers. Note, however, that a similar result would be obtained without modifying the wave breaking term, but assuming that wave breaking is producing an additional flux of spectral energy from high to low wavenumbers. Such downshifting of spectral energy resulting from wave breaking in the presence of modulational instability has been highlighted both theoretically and experimentally, as will be discussed in detail below. In this paper we will show that following this latter approach permits restoring the energy and momentum balance without the need to modify the wave breaking sink term itself.

\subsection{Frequency downshift related to the modulational instability in the presence of wave breaking}

It is well known that a uniform train of Stokes waves is unstable to the so-called modulational instability, as shown theoretically and experimentally by Benjamin and Feir (1967). Following Benjamin and Feir's pioneering work, numerical simulations of the evolution of gravity wave spectra of fairly narrow bandwidth were performed (Alber, 1978; Dysthe et al., 2003), and a number of experimental investigations were conducted on the long-time evolution of nonlinear wave trains (Lake et al., 1977; Melville, 1982; Tulin and Waseda, 1999). The modulational instability is an interaction among three monochromatic wave trains: carrier $\left(\omega_{0}\right)$, upper $\left(\omega_{0}+\delta \omega_{0}\right)$, and lower $\left(\omega_{0}-\delta \omega_{0}\right)$ sideband waves. An asymmetric growth of the sidebands causes the lower to grow at the expense of the upper. In the absence of wave breaking, the evolution occurs in a recurrent fashion, where the modulation periodically increases and decreases, the wave form returning periodically to its previous form. The effect of breaking dissipation is to increase and render irreversible the energy difference of the upper and the lower sidebands after peak modulation. The end state of the evolution following break- ing is an effective downshifting of the spectral energy (Tulin and Waseda, 1999). Although these studies were performed within the framework of monochromatic wave trains, we will assume here that a frequency downshift associated with wave breaking is still occuring in the case of a broad-banded wave spectrum. Since the frequency downshift effectively reduces the wave momentum when the wave energy is conserved, it may provide the mechanism required to solve the problem of imbalance between momentum and energy reported in Sect. 2.

It should be stressed that the modulation instability without wave breaking would produce no downshifting on the long term, and would not be associated with a loss of energy and momentum. In that sense it is a conservative process. Similarly, as reported by Hasselmann et al. (1994), conservation of energy and momentum both apply for all resonant wave-wave interaction processes, including four-wave and three-wave interactions. On the contrary, the process invoked here assumes a further downshifting specifically associated with wave breaking, which violates energy and momentum conservation.

\subsection{New formulation of the wave breaking source term}

Due to the preceding discussion, we will assume henceforth that, in addition to reducing the spectral energy, wave breaking also produces a transfer of energy to lower frequencies. The detailed mechanism for the frequency change is rather unknown. It might be a continuous and gradual process, or result from a superposition of local, abrupt, and discrete processes (Huang et al., 1996). Whatever the detailed process, we will describe the frequency downshift by means of a downward energy flux $\boldsymbol{f}(k, \varphi)$ in the wavenumber space. In a first time calculation, we will assume that $\boldsymbol{f}(k, \varphi)$ is radially oriented in the k-space, and therefore that $f_{\varphi}=0$. As discussed above, the radial component $f_{k}$ should be related to the wave breaking dissipation. As a simplified approach, we will assume that $f_{k}$ is merely proportional to the dissipation term. Dimensional consideration then leads to the following expression of the energy flux:

$\boldsymbol{f}(k, \varphi)=\mu\left(\frac{\partial \Phi}{\partial t}\right)_{\mathrm{br}} \boldsymbol{k}$,

where $\mu$ is a non-dimensional coefficient.

The divergence of this energy flux in the wavenumber space gives rise to a supplementary source term $(\partial \Phi / \partial t)_{\mathrm{S}}$ according to the conservation equation:

$\left(\frac{\partial \Phi}{\partial t}\right)_{\mathrm{S}}=-\operatorname{div}_{k}(\boldsymbol{f})$

where $\operatorname{div}_{k}$ stands for the divergence operator in the wavenumber space. According to Eq. (20), this may be rewritten:

$\left(\frac{\partial \Phi}{\partial t}\right)_{\mathrm{S}}=-\frac{\mu}{k} \frac{\partial}{\partial k}\left[k^{2}\left(\frac{\partial \Phi}{\partial t}\right)_{\mathrm{br}}\right]$, 
or equivalently

$\beta_{\mathrm{S}}=-\frac{\mu}{k \Phi} \frac{\partial}{\partial k}\left(k^{2} \beta_{\mathrm{br}} \Phi\right)$

The energy and momentum budgets can now be performed exactly as in Sect. 2, except that $\beta_{\text {br }}$ should now be replaced by $\beta_{\mathrm{br}}+\beta_{\mathrm{s}}$, where $\beta_{\mathrm{s}}$ is given by Eq. (23). Equations (18a) and (18b) should thus be replaced by

$$
\begin{aligned}
& \int_{0}^{\infty} \int_{-\pi}^{\pi}\left[\beta_{\mathrm{w}}+\beta_{\mathrm{visc}}\right] \Phi k \mathrm{~d} k \mathrm{~d} \varphi \\
& =-\int_{0}^{\infty} \int_{-\pi}^{\pi}\left[\beta_{\mathrm{br}} \Phi-\frac{\mu}{k} \frac{\partial}{\partial k}\left(k^{2} \beta_{\mathrm{br}} \Phi\right)\right] k \mathrm{~d} k \mathrm{~d} \varphi \\
& \int_{0}^{\infty} \int_{-\pi}^{\pi}\left[\beta_{\mathrm{w}}+\beta_{\mathrm{visc}}\right] M k \mathrm{~d} k \mathrm{~d} \varphi \\
& =-\int_{0}^{\infty} \int_{-\pi}^{\pi}\left[\beta_{\mathrm{br}} M-\frac{\mu}{k} \frac{\partial}{\partial k}\left(k^{2} \beta_{\mathrm{br}} M\right)\right] k \mathrm{~d} k \mathrm{~d} \varphi .
\end{aligned}
$$

As done previously (see Eqs. 14a, 16a, or 17), we express $\beta_{\mathrm{br}}$ under the generic form

$\beta_{\mathrm{br}}(k, \varphi)=-\gamma P(k, \varphi)$,

and thus Eqs. (19a) and (19b) should now be replaced by

$$
\gamma_{1}=\frac{\int_{0}^{\infty} \int_{-\pi}^{\pi}\left(\beta_{\mathrm{w}}+\beta_{\text {visc }}\right) \Phi k \mathrm{~d} k \mathrm{~d} \varphi}{\int_{0}^{\infty} \int_{-\pi}^{\pi}\left[P \Phi-\frac{\mu}{k} \frac{\partial}{\partial k}\left(k^{2} P \Phi\right)\right] k \mathrm{~d} k \mathrm{~d} \varphi}
$$

$$
\gamma_{2}=\frac{\int_{0}^{\infty} \int_{-\pi}^{\pi}\left(\beta_{\mathrm{w}}+\beta_{\mathrm{visc}}\right) M k \mathrm{~d} k \mathrm{~d} \varphi}{\int_{0}^{\infty} \int_{-\pi}^{\pi}\left[P M-\frac{\mu}{k} \frac{\partial}{\partial k}\left(k^{2} P M\right)\right] k \mathrm{~d} k \mathrm{~d} \varphi} .
$$

The value of coefficient $\mu$ is then adjusted in such a way that $\gamma_{1}=\gamma_{2}$, thus ensuring that the energy and momentum budgets are balanced.

Although any of the different combinations of wind and wave breaking growth rate models mentioned in Sect. 2 may be used to perform those calculations, henceforth we report only the results obtained using $\beta_{\mathrm{br}}$ from Komen et al. (1984) and $\beta_{\mathrm{w}}$ from WAMDI (1988), since those model functions led to the smallest ratio $\gamma_{2} / \gamma_{1}$ (i.e. smallest energymomentum imbalance) in Tables 1 and 2. Figure 2 displays the value of coefficient $\mu$ adjusted in such a way that $\gamma_{1}=\gamma_{2}$ in Eqs. (26a) and (26b). The adjusted value of $\mu$ is seen to be of the order of 2 to 3 , with only a weak dependence upon

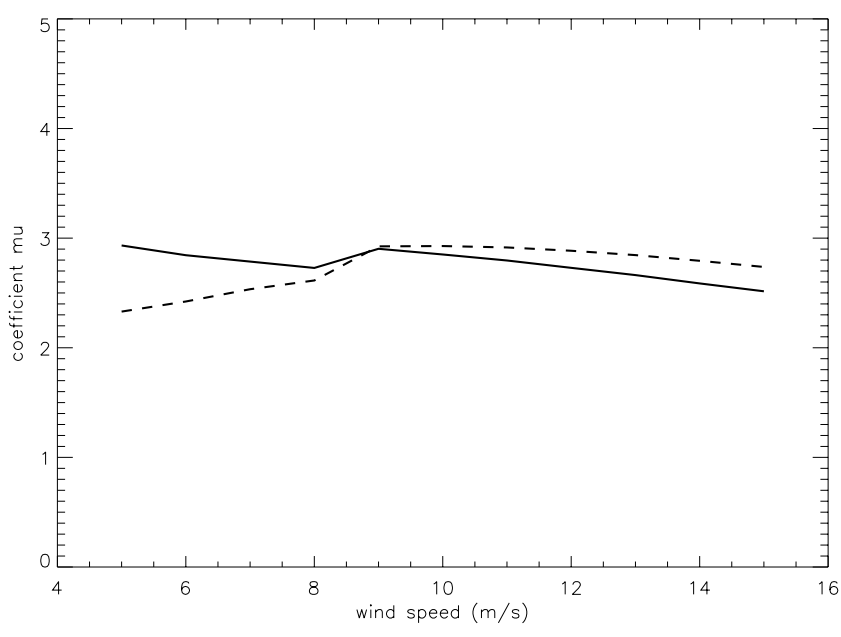

Fig. 2. Coefficient $\mu$ quantifying the downward spectral energy flux (as defined by Eq. 20), allowing balancing of the energy and momentum budgets as a function of wind speed. The wave breaking term $\beta_{b r}$ is from Komen et al. (1984), and the wind input term $\beta_{\mathrm{W}}$ is from WAMDI (1988). The sea wave spectral model for $k<25 k_{\mathrm{p}}$ is from Elfouhaily et al. (1997), with directional spreading function by Resio et al. (2011). Above $k=25 k_{\mathrm{p}}$, the sea wave spectral model is either from Elfouhaily et al. (1997) (solid lines), or from Kudryavtsev et al. (2003) (dashed lines).

wind speed over the $5-15 \mathrm{~m} \mathrm{~s}^{-1}$ range. Considering Eq. (20), one may notice that, since the wave breaking term $(\partial \Phi / \partial t)_{\mathrm{br}}$ is negative, a positive value of $\mu$ implies that the flux $f$ is directed toward decreasing wavenumbers, which is consistent with a frequency downshift. As concerns the high frequency (HF) part of the sea wave spectra, both Elfouhaily et al.'s (1997) and Kudryavtsev et al.'s (2003) models were tested, and both results are displayed in Fig. 2. It can be seen that the value of coefficient $\mu$ is little affected by the choice of the spectral model, except at the lowest wind speeds where $\mu$ tends to be slightly smaller when Kudryavtsev et al.'s HF spectrum is used instead of Elfouhaily et al.'s HF spectrum.

\subsection{Comparison with previous studies on downshifting associated with wave breaking}

The experiments performed by Tulin and Waseda (1999) permitted them to quantify the downward flux associated with wave breaking. Although these experiments were carried out in a laboratory with discrete waves, it may give order of magnitude estimates allowing comparisons with our results.

The evolution of the lower and upper sidebands of a system of discrete waves in presence of wave breaking was studied by Tulin (1996), and is given by equation (5.10) from Tulin and Waseda (1999):

$\frac{\partial}{\partial t}\left(E_{-1}-E_{+1}\right)=\eta D_{\mathrm{b}} /(\delta \omega / \omega)+($ higher harmonic terms $)$.

The influence of the higher harmonic terms is negligible and will be ignored henceforth. $E_{0}, E_{-1}$ and $E_{+1}$ are the energies 
of the wavetrain in the carrier, lower and upper sidebands, with angular velocities $\omega, \omega-\delta \omega, \omega+\delta \omega$, and wavenumbers $k, k-\delta k, k+\delta k$, respectively. $D_{\mathrm{b}}=-\partial E_{3} / \partial t$ is the energy dissipation by wave breaking, where $E_{3}=E_{0}+E_{-1}+E_{+1}$, and $\eta$ is a nondimensional coefficient.

The time derivative of $\left(E_{-1}-E_{+1}\right)$ can also be described as an energy flux $f_{E k}$ in the wavenumber space:

$f_{E k}=-2 \delta k \frac{\partial}{\partial t}\left(E_{-1}-E_{+1}\right)$.

In the domain of gravity waves, the dispersion relation $\omega=$ $\sqrt{g k}$ yields $\delta k / k=2 \delta \omega / \omega$. Therefore, from Eqs. (27) and (28) one gets

$\frac{f_{E k}}{\left(\partial E_{3} / \partial t\right)}=4 \eta k$

Equation (29) expresses the ratio between the k-component of energy flux in wavenumber space and energy dissipation rate due to wave breaking in the case of a discrete wavetrain in the vicinity of wavenumber $k$. Equation (20) proposed a similar relation, but involving this time a broadband spectrum instead of a discrete wavetrain. Equation (20) may be rewritten

$\frac{f_{k}(k, \varphi)}{(\partial \Phi / \partial t)_{\mathrm{br}}}=\mu k$.

Assuming that the results of discrete wavetrains may be extrapolated to a broadband spectrum, consistency between Eqs. (29) and (30) would be obtained by taking $\mu=4 \eta$. Tulin and Waseda's (1999) experimental value of $\eta$ is $\eta=0.4$, while the theoretical estimate by Tulin (1996) is $\eta \approx 0.4$ for a strong breaker to $\eta \approx 0.7$ for a weak breaker. The value of $\mu$ expected to be consistent with those studies would thus be $\mu \approx 1.6$ to 2.8 .

Such a comparison between broadband spectra at sea with studies involving discrete wave systems should not be overinterpreted, and only orders of magnitude estimates are relevant here. It appears, however, that the values that we obtained in Fig. 2 ( $\mu$ between 2 and 3 ) are consistent with the ones expected from Tulin's (1996) and Tulin and Waseda's (1999) studies. It may be noted that when, instead of using $\beta_{\mathrm{br}}$ from Komen et al. (1984) and $\beta_{\mathrm{w}}$ from WAMDI (1988), as done in Fig. 2, one uses $\beta_{\text {br }}$ from Ardhuin et al. (2010) and $\beta_{\mathrm{w}}$ from the JBA model (the combination of source terms resulting from the most recent studies), the required coefficient $\mu$ reaches significantly higher values (between 3 and 6). Also, we will see in Sect. 4 that introducing directional diffusion reduces significantly the value of parameter $\mu$ required.

\section{Assessment of the ability of the model to reproduce the empirically determined sea wave spectrum}

\subsection{Methodology}

The requirement for stationarity of the sea wave spectrum, which is expected for fully developed seas, implies that the source and sink terms balance each other at every wavenumber $k$ and direction $\varphi$. Therefore, for any $k$ and $\varphi$, the total source term $(\partial F / \partial t)_{\mathrm{T}}$ should vanish, which reads

$$
\begin{aligned}
\left(\frac{\partial F(k, \varphi)}{\partial t}\right)_{\mathrm{T}} & =\left(\frac{\partial F}{\partial t}\right)_{\mathrm{w}}+\left(\frac{\partial F}{\partial t}\right)_{\mathrm{visc}}+\left(\frac{\partial F}{\partial t}\right)_{\mathrm{br}} \\
& +\left(\frac{\partial F}{\partial t}\right)_{\mathrm{S}}+\left(\frac{\partial F}{\partial t}\right)_{\mathrm{nl}}=0 .
\end{aligned}
$$

Note that, while in the preceding sections integration of the balance equations over wavenumber space allowed us to ignore the resonant nonlinear interactions that conserve energy and momentum, these need, however, to be included as soon as the balance equation is written at a given wavenumber.

Of course, due to the inaccuracies of the empirically modeled sea wave spectrum, as well as the simplistic nature of the modeled source and sink terms used, one cannot expect Eq. (31) to be fulfilled at every location $(k, \varphi)$ within the spectral plane. At most one can expect $(\partial F(k, \varphi) / \partial t)_{\mathrm{T}}$ to be close to zero on average. In order to assess the efficiency with which the different source terms of Eq. (31) cancel out each other, we define a cost function $K$. On dimensional grounds, this cost function is taken as the average of $1 / \omega\left|\partial\left(k^{4} F(k, \varphi)\right) / \partial t\right|_{\mathrm{T}}$ over the spectral plane. Also, a similar weight is given to the different wavenumber intervals of the sea wave spectrum in logarithmic scale. This leads to the following cost function:

$$
K=\frac{\int_{k=k_{\min }}^{k_{\max }} \int_{-\pi}^{\pi} \frac{1}{\omega}\left|\frac{\partial\left(k^{4} F\right)}{\partial t}\right|_{\mathrm{T}} \mathrm{d}(\ln k) \mathrm{d} \varphi}{\int_{k=k_{\min }}^{k_{\max }} \int_{-\pi}^{\pi} \mathrm{d}(\ln k) \mathrm{d} \varphi} .
$$

The lower limit of the integration is taken as $k_{\min }=0.1 k_{\mathrm{p}}$, where $k_{\mathrm{p}}=g(0.84 / U)^{2}$ is the spectral peak, $g$ is acceleration of gravity and $U$ is the $10 \mathrm{~m}$ wind speed. The determination of the upper limit $k_{\max }$ will be discussed below. While ideally $K$ should be zero, comparison of the values of $K$ obtained with different approaches will allow comparison of the ability of the source terms to reproduce the empirical spectra over the spectral range $\left[k_{\min }, k_{\max }\right]$.

\subsection{Resonant nonlinear term}

The resonant four-wave nonlinear term $(\partial F / \partial t)_{\mathrm{nl}}$ in Eq. (31) was computed by the so-called WRT method based on Webb's (1978) approach, and described in detail by Van Vledder (2006). This method is based upon a number of 
analytical transformations to remove the $\delta$-functions in the Boltzmann integral obtained by Hasselmann (1962).

As the gravity-capillary transition is approached, for wavelengths in the neighborhood of $1.7 \mathrm{~cm}$, another type of resonant nonlinear interactions involving three-wave rather than four-wave resonance conditions occurs (Valenzuela and Laing, 1972). Those three-wave interactions also conserve energy and momentum (Hasselmann et al., 1994). They are not accounted for in this study, and as a consequence that region close to the gravity-capillary transition will not be used to estimate the cost function $K$. The gravity-capillary transition occurs at wavenumber $k_{\mathrm{m}}=(\mathrm{g} / T)^{1 / 2}=364 \mathrm{rad} \mathrm{m}^{-1}$, where $T=74 \times 10^{-6} \mathrm{~m}^{3} \mathrm{~s}^{-2}$ is the surface tension/density ratio for water. In the figures displayed below, the upper limit of integration in Eq. (32) will be taken as $k_{\max }=0.2 k_{\mathrm{m}}$. Even if this choice may seem rather arbitrary, the behavior of $K$ is not qualitatively different if other choices are made for $k_{\max }$ between $0.05 k_{\mathrm{m}}$ and $k_{\mathrm{m}}$, although $K$ would increase by a factor of up to 4 if $k_{\max }=k_{\mathrm{m}}$ rather than $k_{\max }=0.2 k_{\mathrm{m}}$ were used. This is related to the fact that close to $k=k_{\mathrm{m}}$ the spectrum is modified by processes not taken into account in this study, such as three-wave resonant interactions.

\subsection{Comparison with the classical approach}

The values of the cost function K obtained here may be compared to the ones obtained when the frequency downshift introduced above is ignored. In that case, the supplementary term $(\partial F / \partial t)_{\mathrm{S}}$ vanishes. This is the classical way of dealing with wave breaking, and it can be obtained simply by ignoring the momentum balance equation. In that case we just take $\gamma=\gamma_{1}$, as given by the energy Eq. (19a), and we ignore Eq. (19b). In Fig. 3, the cost function $K$ obtained with both approaches is displayed. Again, the wave breaking and wind source terms are taken from Komen et al. (1984) and WAMDI (1988), respectively. As expected, the cost function is seen to increase with wind speed, since the net residual obtained after algebraic summation of the different growth rates scales with the wind input growth rate, and thus increases with wind speed. The version using Kudryavtsev et al.'s (2003) HF spectrum gives systematically higher cost function $K$. In all cases, the cost function obtained with the approach of this paper is smaller than the one obtained with the classical approach, indicating that the formulation of source terms proposed in this paper produces sea wave spectra that are closer to the empirical wave spectra.

Other tests (not shown) were also performed using other model functions for the source terms $\beta_{\mathrm{br}}$ and $\beta_{\mathrm{w}}$. For example, if the most recent model functions involving $\beta_{\mathrm{br}}$ from Ardhuin et al. (2010) and $\beta_{\mathrm{w}}$ from the JBA model are used, then the cost function $K$ obtained with the approach of this paper is virtually identical to the one obtained with the classical approach if we use Kudryavtsev et al. (2003) HF spectrum, and slightly higher than the classical approach if we use Elfouhaily et al. (1997) HF spectrum. This indicates that

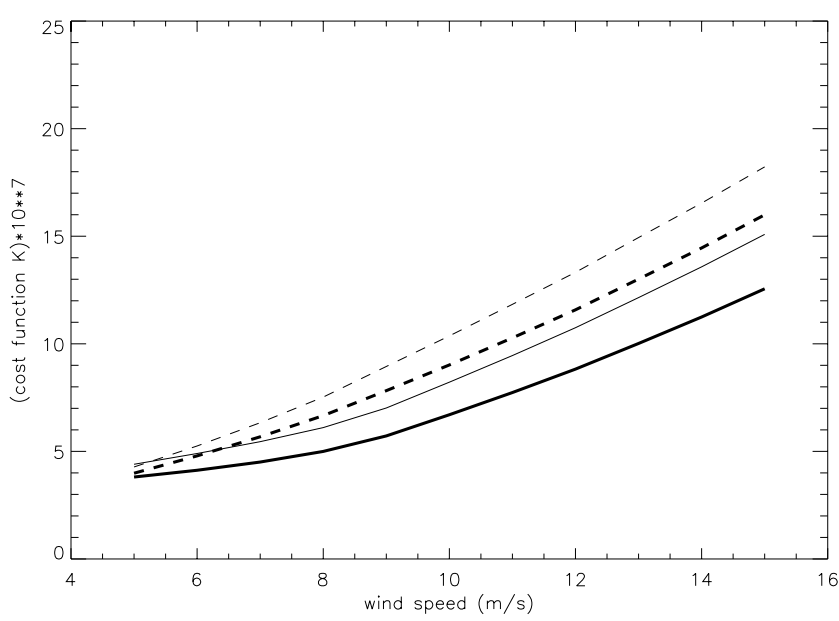

Fig. 3. Cost function $K \times 10^{7}$, as defined by Eq. (32), corresponding to the model of this paper (thick lines), compared with the cost function $\mathrm{K}$ obtained in the classical approach (thin lines), as a function of wind speed. The sea wave spectral models and source terms are the same as in Fig. 2, involving high wavenumber spectrum from Elfouhaily et al. (1997) (solid lines), or from Kudryavtsev et al. (2003) (dashed lines).

using the approach of this paper is less useful if the Ardhuin et al. (2010) and JBA models are used, even though in this latter case the strong energy/momentum imbalance should be addressed somehow.

It may be interesting to illustrate in wavenumber space where the imbalance is occurring. The net variation rate of sea wave momentum may be written

$\left(\frac{\partial M}{\partial t}\right)_{T}=\int g(k) \mathrm{d}(\ln k)$

with

$g(k)=\rho \omega k^{2} \int_{-\pi}^{\pi}\left(\frac{\partial F}{\partial t}\right)_{T} \cos \varphi \mathrm{d} \varphi$.

In Eq. (33b), $(\partial F / \partial t)_{\mathrm{T}}$ includes all the terms of Eq. (31). In a stationary situation the spectrum does not evolve anymore and $g(k)$ should be zero everywhere. Unfortunately, due to the inaccuracies of the models this cannot be achieved perfectly. Figure 4 displays an example of the variation of function $g$ as a function of $\log _{10}(k)$. Due to the definition of function $g(k)$ in Eq. (33a), momentum balance implies that the areas above and below the line $g(k)=0$ should be equal in Fig. 4. By construction this is achieved when the model of this paper is used (full line). When the classical approach is used (dashed line), the fact that we have taken $\gamma=\gamma_{1}$ ensures that the overall energy balance requirement is fulfilled. As concerns the momentum balance, however, it can be seen in Fig. 4 that the area over the line $g(k)=0$ is significantly larger than the area below the line. This indicates that the integrated momentum variation rate is positive, 


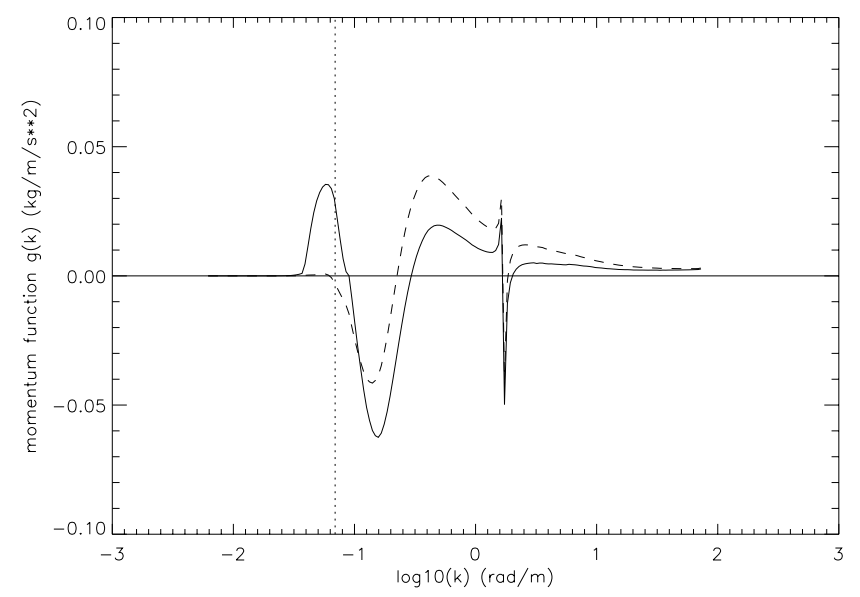

Fig. 4. Momentum function $g(k)$ (Eq. 33a) as a function of $\log _{10}(k)$. Wind speed is taken as $10 \mathrm{~m} \mathrm{~s}^{-1}$. The sea wave spectral model is the same as in Fig. 2, with high wavenumber spectrum from Elfouhaily et al. (1997). The dotted vertical line indicates the position of the spectral peak $k_{\mathrm{p}}$. The wave breaking term $\beta_{\mathrm{br}}$ is from Komen et al. (1984), and the wind input term $\beta_{\mathrm{W}}$ is from WAMDI (1988). Solid line results from the model of this paper. Dashed line results from the classical approach taking $\gamma=\gamma_{1}$. The discontinuity near $\log _{10} k=0.2 \mathrm{rad} \mathrm{m}^{-1}$ is an artefact produced by the change of directional spreading function of the model sea wave spectrum taken at $k=25 k_{\mathrm{p}}$.

leading to unrealistic accumulation of momentum within the wave system, as discussed in Sect. 2.

From Figs. 3 and 4, it appears that there remains some deficiency either in the formulation of source terms or in the empirical spectra used here. I have tested different modifications of the empirical spectrum and source functions in order to improve the quality of the fit as estimated through the cost function $K$. A rather efficient way to further reduce the cost function was obtained by smoothing out the transition between the azimuthal spreading function by Resio et al. (2011) (used at the lower wavenumbers) and the one by Elfouhaily et al. (1997). Moreover, in the standard version of the model, the position of this transition had been chosen rather arbitrarily at $k=k_{\mathrm{o}}=25 k_{\mathrm{p}}$ on the basis that it corresponded to the wavenumber domain where Resio et al.'s model was validated against data (see Sect. 2.2.4 above). It is, however, possible to make $k_{\mathrm{o}}$ an adjustable quantity. For example, for $U=10 \mathrm{~m} \mathrm{~s}^{-1}$ using Elfouhaily et al.'s (1997) HF spectrum and including the aforementioned smoothing, an optimum is found by taking $k_{\mathrm{o}} \approx 90 k_{\mathrm{p}}$ (instead of $k_{\mathrm{o}}=25 k_{\mathrm{p}}$ ). In that optimal case, the cost function $K \times 10^{7}$ is reduced from 6.7 to 6.25 . Other trials were done by modifying the source functions, but they did not lead to very significant improvements.

\subsection{Effect of azimuthal diffusion of spectral energy}

Up to now, the downward energy flux $f(k, \varphi)$ associated with wave breaking was assumed to be radially oriented, and thus its azimuthal component $f_{\varphi}$ was assumed to be equal to zero. In order to relax that constraint, I tested an approach in which, in addition to inward flux, wave breaking was also associated with azimuthal diffusion of the spectral energy. Such azimuthal diffusion should be somehow related to the inward energy flux and its azimuthal gradient. The following expression is chosen here for the azimuthal energy flux:

$f_{\varphi}=v \frac{\partial f_{k}}{\partial \varphi}$,

where the non-dimensional constant $v$ plays the role of a diffusion coefficient.

The supplementary source term $(\partial \Phi / \partial t)_{\mathrm{S}}$ can be computed again from the conservation Eq. (21), in which both radial and azimuthal components of $\boldsymbol{f}$ are now considered, and the resulting values of $\mu$ can thus be obtained for any value of $\nu$. Figure 5 displays the parameter $\mu$ characterizing the strength of inward flux corresponding to those values of diffusion parameter $\nu$. It is seen that $\mu$ decreases with increasing values of $v$, while still remaining positive. For $v$ of the order of 0.5 , the flux parameter $\mu(v)$ is about a factor of 2 smaller than $\mu(0)$. As $v$ increases further, $\mu(v)$ still decreases down to about $(1 / 5) \mu(0)$. However, in any case it is seen that $\mu$ remains positive, which means that a downward flux of spectral energy is still required in order to fulfill simultaneously the balance of both energy and momentum budgets.

\section{Summary and conclusions}

Using a unified two-dimensional sea wave spectral model over the whole range of wavenumbers at full development, I attempted to assess the compatibility of the energy and momentum budgets over the whole spectral range. For fully developed situations among the various combinations of model functions for wave breaking and wind source terms tested, none of them allowed the concurrent fulfillment of the integral energy and momentum balance equations. For example, assuming that the integral energy balance equation is fulfilled, a proportion of about one half or more of the momentum brought by the wind to the waves would accumulate within the wave system. This is in contradiction with the fact that a fully developed sea is expected to be nearly stationary. This indicates that either the chosen spectral shapes are unrealistic, or that the source term parameterizations are unrealistic. In this paper I choose to consider the empirical spectra as the observational reference, and I propose a correction to the source terms, based on physical grounds, permitting to exhibit a solution where the energy and momentum balance is restored. 


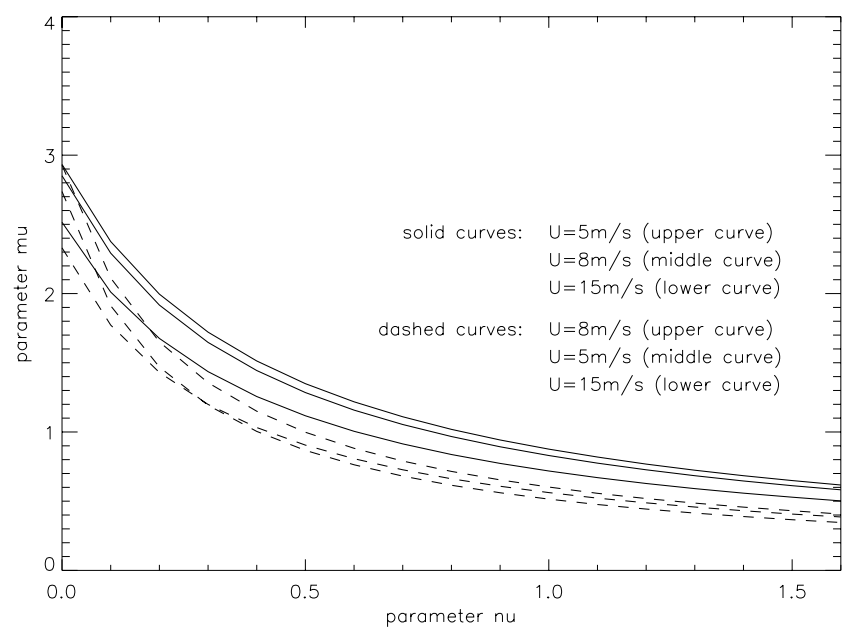

Fig. 5. Spectral flux parameter $\mu$ as a function of diffusion parameter $v$, displayed for 5,8 , and $15 \mathrm{~m} \mathrm{~s}^{-1}$ wind speeds. The sea wave spectral models are the same as in Fig. 1, involving high wavenumber spectrum from Elfouhaily et al. (1997) (solid lines) or from Kudryavtsev et al. (2003) (dashed lines).

The following approach is thus proposed in order to reconcile both energy and momentum budgets. It is known, both experimentally and theoretically, that wave breaking may contribute to frequency downshift of a narrow-banded wave spectrum when the modulational instability is combined with wave breaking. In this paper it is assumed that such a frequency downshift also occurs for the sea wave spectrum, despite the fact that it is a broadbanded spectrum. This is expressed by assuming that, in addition to dissipation, wave breaking is also associated with an energy flux $f$ directed toward low wavenumbers, and quantified by means of a nondimensional coefficient $\mu$ according to Eq. (20). For the model source functions used to obtain the results displayed in this paper, coefficient $\mu$ is estimated to be of the order of 2 to 3 for wind speeds within the $5-15 \mathrm{~m} \mathrm{~s}^{-1}$ range, which scales reasonably well with Tulin and Waseda's (1999) laboratory observations. A cost function $K$ is computed to allow assessment of the source functions ability to reproduce the empirically determined two-dimensional sea wave spectrum at full development. For the model source functions used in this paper, the introduction of this inward spectral flux $f$ improves the consistency between source terms and empirical spectra, as estimated by the cost function $K$. These studies were performed using the wave breaking and wind energy transfer rates by Komen et al. (1984) and WAMDI (1988), respectively, because this combination of source terms produced the smallest energy and momentum budget inconsistency (smallest ratio $\gamma_{2} / \gamma_{1}$ in Table 2). Similar results were obtained by using other models of wave breaking and wind input energy transfer rates, although with some combinations of transfer rate models the approach of this paper gave a nearly identical or slightly higher cost function (i.e. slightly lower con- sistency with empirical spectra) than the classical approach Other tests were performed in which it was assumed that, in addition to inward flux, wave breaking was also associated with azimuthal diffusion of the spectral energy. Incorporating such azimuthal diffusion reduced the inward flux parameter $\mu$ by a factor of up to 5 , although it was not be able to remove entirely the inward flux.

As concerns the sea wave spectral model, two kinds of reference spectra were taken at high wavenumbers $\left(k>25 k_{\mathrm{p}}\right)$ : the spectrum by Elfouhaily et al. (1997), and the one by Kudryavtsev et al. (2003). Although the momentum of waves is particularly sensitive to the directional spectrum at high wavenumbers, the same tendencies were obtained with those two kinds of wavenumber spectra.

Ultimately, this paper shows evidence that, with the wind and wave breaking source terms available in the literature, the integral energy and momentum balance equations cannot be fulfilled concurrently for fully developed seas, which is in contradiction with the fact that a fully developed sea is expected to be nearly stationary. However, the additional process proposed here is able to restore the consistency of energy and momentum budgets.

\section{Appendix A}

\section{JBA model for the atmospheric term for growth rate}

The JBA model mentioned in this paper accounts for the wind input term $\beta_{\mathrm{w}}$ of wave growth rate, as proposed by Janssen's (1991) quasi-linear theory, including tuning modifications by Banner and Morison (2010) and Ardhuin et al. (2010). According to this model, the dimensionless critical height, $\mu_{1}$, is defined as

$$
\begin{aligned}
\mu_{1}(k, \varphi)= & \left(u_{*} / c\right)^{2}\left(g z_{\mathrm{o}} / u_{*}^{2}\right) \\
& \exp \left(J_{1} \kappa /\left[\cos \varphi\left(u_{*} / c+0.006\right)\right]\right),
\end{aligned}
$$

where $\kappa=0.4$ is Van Karman constant, $c$ is the phase speed, and $J_{1}=0.99$. The roughness length $z_{\mathrm{o}}$ is given as

$z_{\mathrm{o}}=\frac{0.01 u_{*}^{2}}{g \sqrt{1-c_{\mathrm{o}}\left(\tau_{\mathrm{w}} / \tau\right)}}$,

where $c_{\mathrm{o}}=0.8$, and the ratio of wave-induced stress $\tau_{\mathrm{w}}$ to the total stress $\tau$ is taken from Janssen's (1989) Eq. (7).

The Miles parameter $b$ is given by

$$
\begin{aligned}
& b(k, \varphi)=J_{2} \mu_{1}\left(\ln \left(\mu_{1}\right)\right)^{4} / \kappa^{2} \text { for } \mu_{1} \leq 1, \text { where } J_{2}=1.6 \\
& b(k, \varphi)=0 \text { for } \mu_{1}>1
\end{aligned}
$$

The spectral growth rate is then

$$
\beta_{\mathrm{w}}(k, \varphi)=\frac{\rho_{\mathrm{a}}}{\rho_{\mathrm{w}}} b(k, \varphi) \omega\left[\frac{u_{*}^{\mathrm{red}}(k)}{c}\right]^{2} \cdot[\max (\cos \varphi, 0)]^{2}
$$


Here, $u_{*}^{\text {red }}$ is the reduced friction velocity, which is felt by wavenumber $k$ as a consequence of the sheltering of the short waves by the longer waves. We express it by removing the integrated waveform drag due to waves with wavenumbers between 0 and $k$, which gives

$$
\begin{gathered}
\left(u_{*}^{\mathrm{red}}\right)^{2}=u_{*}^{2}-s_{\mathrm{u}} \int_{0}^{k} \int_{-\pi}^{\pi} \frac{\rho_{\mathrm{w}}}{\rho_{\mathrm{a}}} \beta_{\mathrm{w}}\left(k^{\prime}, \varphi\right) \omega\left(k^{\prime}\right) F\left(k^{\prime}, \varphi\right) \\
\cos \varphi k^{\prime} \mathrm{d} k^{\prime} \mathrm{d} \varphi,
\end{gathered}
$$

where the sheltering coefficient $s_{\mathrm{u}}$ is taken as $s_{\mathrm{u}}=1$ following Ardhuin et al. (2010).

Equations (A4) and (A5) are solved iteratively.

\section{Appendix B}

\section{Expression of the wave breaking source term by Ardhuin et al. (2010)}

The threshold-based formulation for the wave breaking source term $P_{\mathrm{a}}(k, \varphi)$ introduced in Eq. (17) is decribed by Ardhuin et al. (2010) as the sum of a saturation-based term $P_{\text {sat }}$, a cumulative term $P_{\mathrm{cu}}$, and a term related to waveturbulence interaction $P_{\text {turb }}$, as follows:

$$
P_{\mathrm{a}}(k, \varphi)=P_{\text {sat }}(k, \varphi)+P_{\text {cu }}(k, \varphi)+P_{\text {turb }}(k, \varphi) .
$$

The last term of the right-hand side $P_{\text {turb }}$ due to waveturbulence interactions is expected to be much weaker than both other terms and is neglected by Ardhuin et al. (2010). The saturation-based term $P_{\text {sat }}$ is given as

$$
\begin{aligned}
P_{\text {sat }}(k, \varphi)= & \omega \frac{C_{\mathrm{sat}}}{B_{\mathrm{r}}^{2}}\left\{\delta \cdot \max \left[B_{1}(k)-B_{\mathrm{r}}, 0\right]^{2}+(1-\delta)\right. \\
& \left.\cdot \max \left[B^{\prime}(k, \varphi)-B_{\mathrm{r}}, 0\right]^{2}\right\} .
\end{aligned}
$$

In this expression, $B^{\prime}(k, \varphi)$ is a direction-integrated spectral saturation, with a restriction of the integration over a $\pm \Delta \varphi$ interval (with $\Delta \varphi=80^{\circ}$ ) in order to maintain a dependence of dissipation rate upon direction. It is expressed here as

$B^{\prime}(k, \varphi)=\frac{\int_{\varphi-\Delta \varphi}^{\varphi+\Delta \varphi} \cos ^{s_{\mathrm{B}}}\left(\varphi-\varphi^{\prime}\right) B\left(k, \varphi^{\prime}\right) \mathrm{d} \varphi^{\prime}}{\int_{\varphi-\Delta \varphi}^{\varphi+\Delta \varphi} \cos ^{s_{\mathrm{B}}}\left(\varphi-\varphi^{\prime}\right) \mathrm{d} \varphi^{\prime}}$,

where $B(k, \varphi)=k^{4} F(k, \varphi)$, and

$B_{1}(k)=\max \left[B^{\prime}(k, \varphi), 0 \leq \varphi<2 \pi\right]$.

Following Ardhuin et al. (2010), in Eq. (B2) the threshold for the onset of breaking is taken as $B_{\mathrm{r}}=0.0009$, and the other constants are $C_{\text {sat }}=2.2 \times 10^{-4}, \delta=0$, and $s_{\mathrm{B}}=2$.

The cumulative term $P_{\mathrm{cu}}$ represents the smoothing of the surface by big breakers with celerity $C^{\prime}$ that wipe out smaller waves of phase speed $C$. It is expressed by Ardhuin et al. (2010) as follows:

$$
\begin{gathered}
P_{\mathrm{cu}}(k, \varphi)=-1.44 C_{\mathrm{cu}} \int_{0}^{\mathrm{r}_{\mathrm{cu}} f} \int_{0}^{2 \pi} \max \left[\sqrt{B\left(k^{\prime}, \varphi^{\prime}\right)}-\sqrt{B_{\mathrm{r}}}, 0\right]^{2} \\
\Delta C \mathrm{~d} \varphi^{\prime} \mathrm{d} k^{\prime},
\end{gathered}
$$

where $f$ is sea wave frequency, $\Delta C=\left\|\boldsymbol{C}-\boldsymbol{C}^{\prime}\right\|$, and the constants are taken as $C_{\mathrm{cu}}=-0.4$ and $r_{\mathrm{cu}}=0.5$.

Acknowledgements. The author wishes to thank D. J. Webb and both reviewers for their useful and constructive comments.

Edited by: J. M. Huthnance

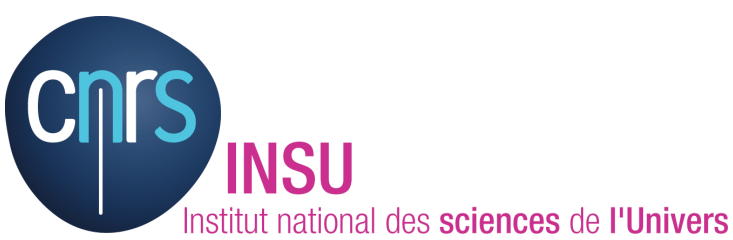

The publication of this article is financed by CNRS-INSU.

\section{References}

Alber, I. E.: The effect of randomness on the stability of twodimensional surface wavetrains, P. Roy. Soc. Lond. Ser. A, 363, 525-546, 1978.

Ardhuin, F., Chapron, B., and Collard, F.: Observation of swell dissipation across oceans, Geophys. Res. Lett., 36, L06607, doi:10.1029/2008GL037030, 2009.

Ardhuin, F., Rogers, E., Babanin, A. V., Filipot, J. F., Magne, R., Roland, A., van der Westhuysen, A., Queffeulou, P., Lefevre, J. M., Aouf, L., and Collard, F.: Semi-empirical Dissipation Source Functions for Ocean Waves, Part I: Definition, Calibration, and Validation, J. Phys. Oceanogr., 40, 1917-1941, 2010.

Banner, M.: Equilibrium spectra of wind waves, J. Phys. Oceanogr., 20, 966-984, 1990.

Banner, M. L. and Morison, R. P.: Refined source terms in wind wave models with explicit wave breaking prediction, Part I: Model framework and validation against field data, Ocean Model., 33, 177-189, 2010.

Banner, M. L., Jones, I. S. F., and Trinder, J. C.: Wavenumber spectra of short gravity waves, J. Fluid Mech., 198, 321-344, 1989.

Banner, M. L., Gemmrich, J. R., and Farmer, D. M.: Multiscale measurements of ocean wave breaking probability, J. Phys. Oceanogr., 32, 3364-3375, 2002.

Belcher, S. E. and Hunt, J. C. R.: Turbulent shear flow over slowly moving waves, J. Fluid Mech., 251, 109-148, 1993.

Benjamin, T. B. and Feir, J. E.: The disintegration of wave trains on deep water. Part 1. Theory, J. Fluid Mech. 27, 417-430, 1967.

Cox, C. and Munk, W.: Measurement of the roughness of the sea surface from photographs of the Sun's glitter, J. Opt. Soc. Am., 44, 838-850, 1954. 
Donelan, M. A. and Pierson, Jr., W. J.: Radar scattering and equilibrium ranges in wind-generated waves with application to scatterometry, J. Geophys. Res., 92, 4971-5029, 1987.

Donelan, M. A., Hamilton, J., and Hui, W. H.: Directional spectra of wind generated waves, P. T. Roy. Soc. Lond. Ser. A, 315, 509$562,1985$.

Dore, B. D.: Some effects of the air-water interface on gravity waves, Geophys. Astrophys. Fluid Dynam., 10, 215-230, 1978.

Dysthe, K. B., Trulsen, K., Krogstad, H. E., and Socquet-Juglard, H.: Evolution of a narrow-band spectrum of random surface gravity waves, J. Fluid Mech., 478, 1-10, 2003.

Elfouhaily, T., Chapron, B., Katsaros, K., and Vandemark, D.: A unified directional spectrum for long and short wind-driven waves, J. Geophys. Res., 102, 15781-15796, 1997.

Gelci, R., Cazalé, H., and Vassal, J.: Prévision de la houle. La méthode des densités spectroangulaires, Bull. Infor. Comité Central Oceanogr. D’Etude Côtes, 9, 416-435, 1957.

Hara, T. and Belcher, S. E.: Wind forcing in the equilibrium range of wind-wave spectra, J. Fluid Mech., 470, 223-245, 2002.

Hasselmann, K.: On the nonlinear energy transfer in a gravity-wave spectrum, Part 1: General theory, J. Fluid Mech., 12, 481-500, 1962.

Hasselmann, K.: On the nonlinear energy transfer in a gravity-wave spectrum, Part 2: Conservation theorems; wave-particle analogy; irreversibility, J. Fluid Mech., 15, 273-281, 1963.

Hasselmann, K.: On the spectral dissipation of ocean waves due to white capping, Bound.-Lay. Meteorol., 6, 107-127, 1974.

Hasselmann, K., Janssen, P. A. E. M., and Komen, G. J.: II, Wavewave interaction, in Dynamics and Modeling of Ocean Waves, edited by: Komen, G. J., Cavaleri, L., Donelan, M., Hasselmann, K., Hasselmann, S., and Janssen, P. A. E. M., 113-143, Cambridge University Press, New York, 1994.

Hauser, D., Caudal, G., Guimbard, S., and Mouche A. A.: A study of the slope probability density function of the ocean waves from radar observations, J. Geophys. Res., 113, C02006, doi:10.1029/2007JC004264, 2008.

Huang, N. E., Long, S. R., and Shen, Z.: The mechanism for frequency downshift in nonlinear wave evolution, Adv. Appl. Mech., 32, 59-117, 1996.

Jähne, B. and Riemer, K. S.: Two-dimensional wave number spectra of small-scale water surface waves, J. Geophys. Res., 95, 1153111546, 1990.

Janssen, P. A. E. M.: Wave-induced stress and the drag of air flow over sea waves, J. Phys. Oceanogr., 19, 745-754, 1989.

Janssen, P. A. E. M.: Quasi-linear theory of wind-wave generation applied to wave forecasting, J. Phys. Oceanogr., 21, 1631-1642, 1991.

Komen, G. J., Hasselmann, K., and Hasselmann, S.: On the existence of a fully developed wind-sea spectrum, J. Phys. Oceanogr., 14, 1271-1285, 1984.
Kudryavtsev, V., Hauser, D., Caudal, G., and Chapron, B.: A semiempirical model of the normalized radar cross-section of the sea surface, 1, Background model, J. Geophys. Res., 108, 8054, doi:10.1029/2001JC001003, 2003.

Kukulka, T. and Hara, T.: Momentum flux budget analysis of wind-driven air-water interfaces, J. Geophys. Res., 110, C12020, doi:10.1029/2004JC002844, 2005.

Lake, B. M., Yuen, H. C., Rungaldier, H., and Ferguson, W. E.: Nonlinear deep-water waves: theory and experiment, Part 2. Evolution of a continuous wave train, J. Fluid Mech., 83, 49-74, 1977.

Lamb, H.: Hydrodynamics, Cambridge Univ. Press, New York, 1932.

Long, C. E. and Resio, D. T.: Wind wave spectral observations in Currituck Sound, North Carolina, J. Geophys. Res., 112, C05001, doi:10.1029/2006JC003835, 2007.

Melville, W. K.: The instability and breaking of deep-water waves, J. Fluid Mech., 115, 165-185, 1982.

Phillips, O. M.: Spectral and statistical properties of the equilibrium range in wind-generated gravity waves, J. Fluid Mech., 156, 505531, 1985.

Plant, W. J.: A relationship between wind stress and wave slope, J. Geophys. Res., 87, 1961-1967, 1982.

Resio, D. T., Long, C. E., and Perrie, W.: The role of nonlinear momentum fluxes on the evolution of directional wind-wave spectra, J. Phys. Oceanogr., 41, 781-801, 2011.

Snyder, R. L., Dobson, F. W., Elliott, J. A., and Long, R. B.: Array measurements of atmospheric pressure fluctuations above surface gravity waves, J. Fluid Mech., 102, 1-59, 1981.

Tolman, H. L.: Testing of WAVEWATCH-III version 2.22 in NCEP's NWW3 ocean wave model suite, Tech. Rep., NOAA/NWS/NCEP/NMAB, OMB contribution number 214, 2002.

Tulin, M. P.: Breaking of ocean waves and downshifting, in: Waves and nonlinear processes in hydrodynamics, edited by: Grue, J., Gjevik, B., and Weber, J. E., 177-190, 1996.

Tulin, M. P. and Waseda, T.: Laboratory observations of wave group evolution, including breaking effects, J. Fluid Mech., 378, 197232, 1999.

Valenzuela, G. R. and Laing, M. B.: Nonlinear energy transfer in gravity-capillary wave spectra, with applications, J. Fluid Mech., 54, 507-520, 1972.

Van Vledder, G. P.: The WRT method for the computation of nonlinear four-wave interactions in discrete spectral wave models, Coastal Engineering, 53, 223-242, 2006.

WAMDI group: The WAM model - A third generation ocean wave prediction model, J. Phys. Oceanogr., 18, 1775-1810, 1988.

Webb, D. J.: Nonlinear transfer between sea waves, Deep-Sea Res., 25, 279-298, 1978.

Young, I. R., Verhagen, L. A., and Banner, M. L.: A note on the bimodal directional spreading of fetch-limited wind waves, J. Geophys. Res., 100, 773-778, 1995. 